\title{
An integrability result for L-p-vector fields in the plane
}

\section{Journal Article}

Author(s):

Petrache, Mircea

Publication date:

2013-07

Permanent link:

https://doi.org/10.3929/ethz-b-000408632

Rights / license:

In Copyright - Non-Commercial Use Permitted

Originally published in:

Advances in Calculus of Variations 6(3), https://doi.org/10.1515/acv-2012-0102 


\title{
An integrability result for $L^{p}$-vector fields in the plane
}

\author{
Mircea Petrache \\ Communicated by Giuseppe Mingione
}

\begin{abstract}
We prove that if $p>1$, then the divergence of an $L^{p}$-vector field $V$ on a 2-dimensional domain $\Omega$ is the boundary of an integral 1-current if and only if $V$ can be represented as the rotated gradient $\nabla^{\perp} u$ for a $W^{1, p}$-map $u: \Omega \rightarrow S^{1}$. Such a result extends to exponents $p>1$ the result on distributional Jacobians of Alberti, Baldo, Orlandi (2003).
\end{abstract}

Keywords. Vector fields with integer fluxes, topological singularities, distributional Jacobian, Sobolev maps between manifolds.

2010 Mathematics Subject Classification. Primary 35C99, 46E35; secondary 46E40, 53C65, 49Q15, 26B10.

\section{Introduction}

Consider a vector field $V \in L^{p}\left(B^{2}, \mathbb{R}^{2}\right)$. If $\operatorname{div} V=0$, then by the Poincaré Lemma we know that there exists a $W^{1, p}$-function $\psi$ with

$$
V=\nabla^{\perp} \psi
$$

The next case in which the situation is relatively standard is when (in the sense of distributions)

$$
\operatorname{div} V=2 \pi \sum_{i=1}^{N} n_{i} \delta_{x_{i}}, \quad \text { for some } n_{i} \in \mathbb{Z} \backslash\{0\} \text { and } x_{i} \in B^{2} .
$$

Note that we cannot have $V \in L^{p}$ unless $p<2$ holds (consider the model case $V(x)=\frac{x}{|x|^{2}}$, corresponding to $N=1, x_{1}=(0,0), n_{1}=1$ in (1.2)).

The representation (1.1) holds then just locally outside the points $x_{i}$, and the local representations do not lift to a global one. If $p \geq 1$ then we obtain that the function $\psi$ is locally harmonic and $V$ is locally holomorphic. Therefore, it is possible to find a representation of the form (1.1) for a function $\psi \in W^{1, p}\left(B^{2}, \mathbb{R} / 2 \pi \mathbb{Z}\right)$, by taking $\psi=\operatorname{Arg}(V)+C$ for any constant $C$. Equivalently, one could use the 
Green function for the laplacian to obtain a harmonic solution of $\nabla g=V$, and then from the regularity of $g$ the existence of $\psi$ would follow.

If we now consider the preimage $\psi^{-1}(y)$ of any regular value $y \in \mathbb{R} / 2 \pi \mathbb{Z}$ of $\psi$, then we see by Sard's theorem that this will be a rectifiable set, and with the orientation corresponding to the vector field $\nabla g$, we can also consider this set as an integral current $I_{\psi}$ on $\bar{B}^{2}$. The boundary of this current is precisely the sum of Dirac masses in (1.2) (without the " $2 \pi$ " factor):

$$
\partial I_{\psi\llcorner} B^{2}=\sum_{i=1}^{N} n_{i} \delta_{x_{i}}=\frac{1}{2 \pi} \operatorname{div} V .
$$

When passing to the case where we allow $N=\infty$ in (1.2), we have to face the new difficulty that not all the formal infinite sums of Dirac masses can be represented as the distributional divergence of an $L^{p}$-vector field. The most obvious restriction (depending on the Fubini Theorem) is seen as follows. Let $\Sigma$ be a closed smooth Jordan curve and consider its perturbations $\Sigma(t), t \in[-\varepsilon, \varepsilon]$, via a family of diffeomorphisms. Then the flux $f(t)$ of $V$ through $\Sigma(t)$ should satisfy again $f \in L^{p}([-\varepsilon, \varepsilon])$. In particular, it cannot happen that the algebraic sum of the Dirac masses inside $\Sigma$ stays infinite for a set of times $t$ of positive measure.

If we assume for a moment that a rectifiable 1-current $I$ as in (1.3) exists, the above condition would translate by saying that the mass of the slice of $I$ along $\Sigma(t)$ is an $L^{p}$-function of $t$.

In this work we prove a necessary and sufficient condition for a representability property like (1.1) to hold. Consider a smooth domain $\Omega \subset \mathbb{R}^{2}$ or the domain $\Omega=S^{2} \simeq \mathbb{C} \cup\{\infty\}$. Our main result is then:

Main Theorem 1 (first version). Suppose we have a vector field $V \in L^{p}\left(\Omega, \mathbb{R}^{2}\right)$ with $p>1$ whose divergence can be represented by the boundary of an integral 1-current I on $\Omega$, i.e.

$$
\frac{1}{2 \pi} \int V \cdot \nabla \phi=\langle I, d \phi\rangle \quad \forall \phi \in C_{c}^{\infty}(\Omega) .
$$

Then there exists $a W^{1, p}$-function $u: \Omega \rightarrow \mathbb{R} / 2 \pi \mathbb{Z}$ such that $V=\nabla^{\perp} u$ and $\left.u\right|_{\partial \Omega}$ has zero degree. Viceversa, for any $u \in W^{1, p}(\Omega, \mathbb{R} / 2 \pi \mathbb{Z})$ with $\operatorname{deg}\left(\left.u\right|_{\partial \Omega}\right)=0$, the vector field $\nabla^{\perp} u$ belongs to $L^{p}$ and has divergence equal to the boundary of a current in $\Omega_{1}(\Omega)$, in the sense of (1.4).

The zero degree condition on $\partial \Omega$ in the above theorem can be removed in the following way. Consider a $L^{p}$-vector field $V$ such that

$$
\frac{1}{2 \pi} \operatorname{div} V=\partial I+\sum_{i=1}^{N} n_{i} \delta_{x_{i}} \quad \text { for some } n_{i} \in \mathbb{Z} \backslash\{0\} \text { and } x_{i} \in \Omega \text {. }
$$


Then we can find, via the Green function method sketched in the introduction, a vector field $V^{\prime}$ satisfying (1.2) and a function $\psi^{\prime} \in W^{1, p}(\Omega, \mathbb{R} / 2 \pi \mathbb{Z})$ satisfying (1.1), with

$$
\begin{aligned}
\operatorname{deg}\left(\left.\psi^{\prime}\right|_{\partial \Omega}\right) & =\sum_{i=1}^{N} n_{i}, \\
\frac{1}{2 \pi} \operatorname{div}\left(V-V^{\prime}\right) & =\partial I,
\end{aligned}
$$

and we can apply the Main Theorem to the vector field $V-V^{\prime}$ obtaining a function $\psi \in W^{1, p}(\Omega, \mathbb{R} / 2 \pi \mathbb{Z})$ with degree zero on the boundary $\partial \Omega$ and which satisfies $\nabla^{\perp} \psi=V-V^{\prime}$. Then $\psi+\psi^{\prime}$ will satisfy

$$
\begin{aligned}
\nabla^{\perp}\left(\psi+\psi^{\prime}\right) & =V, \\
\operatorname{deg}\left(\left.\left(\psi+\psi^{\prime}\right)\right|_{\partial \Omega}\right) & =\sum_{i=1}^{N} n_{i} .
\end{aligned}
$$

With this construction we obtain the following generalization:

Corollary 1.1. Suppose we have an $L^{p}$-vector field $V$ satisfying (1.5). Then there exists a $W^{1, p}$-function $u: \Omega \rightarrow \mathbb{R} / 2 \pi \mathbb{Z}$ such that $V=\nabla^{\perp} u$ and $\left.u\right|_{\partial \Omega}$ has degree $\sum_{i=1}^{N} n_{i}$. Viceversa, for any $u \in W^{1, p}(\Omega, \mathbb{R} / 2 \pi \mathbb{Z})$ with $\operatorname{deg}\left(\left.u\right|_{\partial \Omega}\right)=d \in \mathbb{Z}$, the vector field $\nabla^{\perp} u$ belongs to $L^{p}$ and satisfies (1.5), where $d=\sum n_{i}$.

In the case $p=1$, a result similar to the Main Theorem above is a subcase of the result of [1]. An equivalent statement of such a result is (see also Section 1.1, where different notations are proposed):

Proposition 1.2 ([1]). For each integral 1-current I of finite mass on $\Omega$ there exists a map $\psi \in W^{1,1}(\Omega, \mathbb{R} / 2 \pi \mathbb{Z})$ such that (in the sense of distributions)

$$
\partial I=\frac{1}{2 \pi} \operatorname{div}\left(\nabla^{\perp} \psi\right) .
$$

The distribution $\operatorname{div}\left(\nabla^{\perp} \psi\right)$ is called distributional Jacobian of $\psi$.

Remark 1.3. As seen in Example 6.1, for $p>1$, unlike the case $p=1$, a large subclass of the boundaries of integral currents is not realized as distributional Jacobian of maps in $W^{1, p}\left(B^{2}, S^{1}\right)$, therefore we must ask for a higher integrability condition for the current $I$ : this is why the existence of the $L^{p}$-vector field $V$ is imposed. 


\subsection{Different formulations of the Main Theorem}

We have at least three ways of looking at the manifold $S^{1}$, namely:

(i) as a subset of $\mathbb{R}^{2}: S^{1}=\left\{(x, y) \in \mathbb{R}^{2}: x^{2}+y^{2}=1\right\}$,

(ii) via a parametrization: $S^{1}=\{(\cos (t), \sin (t)): t \in \mathbb{R}\}$,

(iii) as a group quotient: $S^{1}=\mathbb{R} / 2 \pi \mathbb{Z}$.

When considering $W^{1, p}$-maps on $B^{2}$ with values in $S^{1}$, these three points of view lead to three possible spaces:

(i) $W_{1}=\left\{u \in W^{1, p}\left(B^{2}, \mathbb{R}^{2}\right): u_{1}^{2}(x)+u_{2}^{2}(x)=1\right.$, a.e. $\left.x \in B^{2}\right\}$, which is just the usual definition of $W^{1, p}\left(B^{2}, S^{1}\right)$,

(ii) $W_{2}=\left\{(\cos (\psi), \sin (\psi)): \psi \in W^{1, p}\left(B^{2}, \mathbb{R}\right)\right\}$,

(iii) $W_{3}=\left\{u \in W^{1, p}\left(B^{2}, \mathbb{R}\right)\right\} / \sim$, where $u_{1} \sim u_{2}$ if $u_{1}-u_{2}$ is a measurable map with values on $2 \pi \mathbb{Z}$ a.e. We denote this space by $W^{1, p}\left(B^{2}, \mathbb{R} / 2 \pi \mathbb{Z}\right)$.

The space $W_{1}$ is isomorphic as a (topological vector space) to $W_{3}$ via the diffeomorphism

$$
\phi: \mathbb{R} / 2 \pi \mathbb{Z} \rightarrow S^{1}, \quad t \mapsto(\cos (t), \sin (t)) .
$$

On the other hand, the space $W_{2}$ is different from $W_{1}, W_{3}$ because of the following result:

Theorem 1.4 ([8]). If $1 \leq p<2$ and $u \in W^{1, p}\left(B^{n}, S^{1}\right)$, then the following statements are equivalent:

- $u$ can be strongly approximated by smooth maps $u_{k} \in C^{\infty}\left(B^{n}, S^{1}\right)$.

- $d\left(u^{*} \theta\right)=0$ in the sense of distributions.

- There exists $\tilde{u} \in W^{1, p}\left(B^{n}, \mathbb{R}\right)$ such that $u=(\cos (\tilde{u}), \sin (\tilde{u}))$.

In our work, the space $W_{3}$ seems notationally lighter, but since $W_{1}$ is more common, we would like to reformulate the Main Theorem here:

Main Theorem 2 (second version). Let $V \in L^{p}\left(\Omega, \mathbb{R}^{2}\right)$ with $p>1$ be a vector field satisfying equation (1.4) for an integral 1-current $I$. Then there exists a map $u \in W^{1, p}\left(\Omega, S^{1}\right)$ with degree zero on $\partial \Omega$ such that $V=u_{2} \nabla^{\perp} u_{1}-u_{1} \nabla^{\perp} u_{2}$. Viceversa, for any map $u \in W^{1, p}\left(\Omega, S^{1}\right)$ with zero degree on the boundary, the vector field $u_{2} \nabla^{\perp} u_{1}-u_{1} \nabla^{\perp} u_{2}$ is in $L^{p}$ and has divergence equal to the boundary of an integral current.

We describe how to pass from the first to the second version of the Main Theorem in Section 3.1. 
Our result can be reformulated in somewhat more geometrical terms by identifying differential forms $\alpha \in L^{p}\left(\Omega, \wedge^{1} \Omega\right)$ with vector fields ${ }^{1} V_{\alpha} \in L^{p}\left(\Omega, \mathbb{R}^{2}\right)$ by setting $V_{\alpha}=\left(\alpha_{2},-\alpha_{1}\right)$ if $\alpha=\alpha_{1} d x+\alpha_{2} d y$, so that $d \alpha$ corresponds to $\operatorname{div} V_{\alpha}$. We also observe that if we consider the tangent space of $S^{1}=\mathbb{R} / 2 \pi \mathbb{Z}$ to be identified with $\mathbb{R}$ in the canonical way, then $V_{u^{*} \theta}$ can be identified with $\nabla^{\perp} u$. We obtain therefore the following alternative formulation:

Main Theorem 3 (third version). Let $p>1$, let $\Omega$ be either a regular open domain in $\mathbb{R}^{2}$ or the sphere $S^{2}$, and let $\theta$ be the volume form of $S^{1}$. Then the following equality holds:

$$
\begin{aligned}
& \left\{u^{*} \theta: u \in W^{1, p}\left(\Omega, S^{1}\right), \operatorname{deg}\left(\left.u\right|_{\partial \Omega}\right)=0\right\} \\
& =\left\{\alpha: \alpha \in L^{p}\left(\Omega, \wedge^{1} \mathbb{R}^{2}\right), \exists I \in \mathcal{\ell}_{1}(\Omega),[d \alpha]=\partial I\right\},
\end{aligned}
$$

where $d_{1}(\Omega)$ represents the finite mass integral rectifiable 1 -currents on $\Omega$ and $[d \alpha]$ is the distribution associated to d $\alpha$ by imposing

$$
\langle[d \alpha], \varphi\rangle=\int_{\Omega} d \alpha \wedge \varphi \quad \forall \varphi \in \mathscr{D}_{0}(\Omega) .
$$

\subsection{Ingredients of the proof}

The proof of the first part of our theorem follows from a density result: We prove that the class of $L^{p}$-vector fields with finitely many topological singularities is dense in the class of vector fields satisfying the condition (1.4). This fact is proved in Section 2, and the proof is in the spirit of the work [3] of Bethuel (see also $[2,4,6,10]$ for related results), and is inspired by the ideas present in [12] and in [11]. It is easy to prove the first part of the Main Theorem for $V$ having finitely many singularities. We can then pass to the limit the $W^{1, p}$-maps $u_{k}$ obtained in the simpler case for an approximating sequence

$$
V_{k} \stackrel{L^{p}}{\longrightarrow} V
$$

in order to achieve the representation result in the first part of the Main Theorem (see Section 3).

The second part of the theorem is a direct consequence of a coarea formula (see for example [13]), which is related to the Sard theorem for Sobolev spaces (for which see among others $[5,7,9])$. We state here just the result that we need.

${ }^{1}$ This is a special instance of the identification of $k$-covectors $\alpha$ with $(n-k)$-vectors $V$ in an $n$-dimensional oriented manifold $M$ given by imposing

$$
\langle\beta, V\rangle=\langle\beta \wedge \alpha, \vec{M}\rangle
$$

for all ( $n-k)$-covectors $\beta$, where $\vec{M}$ is an orienting $n$-vector field of $M$. 
Theorem 1.5. If $f \in W_{\mathrm{loc}}^{1, p}\left(M^{m}, N^{n}\right)$ for some manifolds $M, N$, then there exists a Borel representative of $f$ such that $f^{-1}(y)$ is countably $(m-n)$-rectifiable and has finite $\mathscr{H}^{m-n}$-measure for almost all $y \in N$ and such that for every measurable function $g$ there holds

$$
\int_{M} g(x)\left|J_{f}(x)\right| d \mathscr{H}^{m}(x)=\int_{N}\left(\int_{f^{-1}(y)} g(x) d \mathscr{H}^{m-n}(x)\right) d \mathscr{H}^{n}(y),
$$

where $\left|J_{f}(x)\right|=\sqrt{\operatorname{det}\left(D f_{x} \cdot D f_{x}^{T}\right)}$.

\section{A density result}

We consider two classes of vector fields:

$$
\mathcal{V}_{\mathbb{Z}}:=\left\{V \in L^{p}\left(D, \mathbb{R}^{2}\right):(1.4) \text { holds }\right\},
$$

and

$$
\mathcal{V}_{R}:=\left\{V \in \mathcal{V}_{\mathbb{Z}}: V \text { is smooth outside a finite set } S \subset D\right\} .
$$

Since $\mathcal{V}_{\mathbb{Z}}$ is closed in $L^{p}$, it is clear that $\overline{\mathcal{V}}_{R}^{L^{p}} \subset \mathcal{V}_{\mathbb{Z}}$. We want to prove the following result:

Proposition 2.1. With the above notations, $\overline{\mathcal{V}}_{R}{ }^{p^{p}}=\mathcal{V}_{\mathbb{Z}}$ holds.

By the remarks about $\mathcal{V}_{R}$ and $\mathcal{V}_{\mathbb{Z}}$, we just have to prove that any $V \in \mathcal{V}_{\mathbb{Z}}$ can be approximated up to an arbitrary small error $\varepsilon>0$ in $L^{p}$-norm, by some $V_{\varepsilon} \in \mathcal{V}_{R}$. The strategy of our proof is to choose first a "grid of circles of radius $r$ ", on which we mollify appropriately $V$, and then to extend the mollified vector field inside each circle by creating finitely many singularities (note that the number of singularities might become unbounded for $r \rightarrow 0$ ), and by staying $L^{p}$-near the initial $V$. Finally, we will patch together the extensions on each of the balls bounded by these circles, obtaining the wanted approximant $V_{\varepsilon}$. The way in which we "fill the $r$-balls" will be by either radial or harmonic extension: we decide the method to apply depending on the degree of $V_{m}$ on the respective ball (we are guided in this by the result of Demengel [8] cited in Theorem 1.4).

\subsection{Choice of a good covering}

Lemma 2.2. Given a real $r>0$, there exists a natural number $N$, a set of centers $\left\{x_{1}, \ldots, x_{N}\right\}$ and a positive measure subset $E \subset[3 / 4 r, r]^{N}$ such that for all $\left(r_{1}, \ldots, r_{N}\right) \in E$ the following hold:

- The balls $\left\{B_{1}, \ldots, B_{N}\right\}$ with $B_{i}=B_{r_{i}}\left(x_{i}\right)$ cover $B^{2}$.

- The smaller balls $B_{\frac{3}{8}} r_{i}\left(x_{i}\right)$ are disjoint. 
- For some constant depending only on $p$ and on the dimension, there holds

$$
\sum_{i=1}^{N} \int_{\partial B_{i}}\left|V \cdot n_{B_{i}}\right|^{p} d x \leq C_{2, p} r^{-1}\|V\|_{L^{p}\left(B^{2}\right)}^{p},
$$

where $n_{B_{i}}$ is the outer normal to the ball $B_{i}$.

Proof. See Section 4.

The next lemma is needed in order to translate properties of the current $I$ to the vector field $V$.

Lemma 2.3. Given a piecewise smooth domain $\Omega \subset B^{2}$, for almost all $t \in[-\varepsilon, \varepsilon]$ the following properties hold:

- The slice $\left\langle I, \operatorname{dist}_{\partial \Omega}, t\right\rangle$ exists and is a rectifiable 0-current with multiplicity in $2 \pi \mathbb{Z}$.

- The map $\int_{\partial \Omega_{t}} V(y) \cdot n_{t}(y) d \mathscr{H}^{1}(y)$ (where $n_{t}$ is the unit normal to $\left.\partial \Omega_{t}\right)$ is well-defined and coincides with the number $\left\langle I, \operatorname{dist}_{\partial \Omega}, t\right\rangle(1) \in 2 \pi \mathbb{Z}$.

Proof. See Section 5.

Combining the Lemmas 2.2 and 2.3, we obtain:

Lemma 2.4. Given $r>0$, there exists a set of balls $\left\{B_{1}, \ldots, B_{N}\right\}$ with radii in $[3 / 4 r, r]$ such that the thesis of Lemma 2.2 holds and that for any $\Omega$ which is the closure of a connected component of $B^{2} \backslash \bigcup_{i=1}^{N} \partial B_{i}$ the slice $\left\langle I\right.$, dist $\left.\operatorname{din}_{2}, 0\right\rangle$ exists, is a rectifiable 0 -current with multiplicity in $2 \pi \mathbb{Z}$ and

$$
\left\langle I, \operatorname{dist}_{\partial \Omega}, 0\right\rangle(1)=\int_{\partial \Omega} V(y) \cdot n_{\Omega}(y) d \mathscr{H}^{1}(y) \in 2 \pi \mathbb{Z} .
$$

Proof. We can use Lemma 2.2 first, obtaining a set $E \subset[3 / 4 r, r]^{N}$. For a cover $\left\{B_{1}^{\prime}, \ldots, B_{N}^{\prime}\right\}$ corresponding to a density point of $E$, we can then apply Lemma 2.3 for all the closures of connected components of $B^{2} \backslash \bigcup \partial B_{i}^{\prime}$, and then consider the slices for $t \leq 0$ only.

\subsection{Mollification on the boundary and estimates on good and bad balls}

Lemma 2.5. For a choice of balls $B_{i}$ as in Lemma 2.4, it is possible to find a vector field $V_{m} \in C^{\infty}\left(\bigcup_{i} \partial B_{i}, \mathbb{R}^{2}\right)$ such that for all the regions $\Omega$ as in Lemma 2.4 there holds

$$
\begin{aligned}
\forall i, \quad \int_{\partial \Omega} V_{m} \cdot n_{\Omega} d \mathscr{H}^{1} & =\int_{\partial \Omega} V \cdot n_{\Omega} d \mathscr{H}^{1} \in 2 \pi \mathbb{Z}, \\
\left\|V_{m}-V\right\|_{L^{p}\left(\cup_{i} \partial B_{i}\right)} & \leq \varepsilon_{m} .
\end{aligned}
$$


Proof. It is enough to find $V_{m}$ satisfying (2.2) and (2.3), and defined only on the set $\bigcup_{i} \partial B_{i} \backslash\left\{x: \exists i \neq j, x \in \partial B_{i} \cap \partial B_{j}\right\}:=\bigcup_{i} \partial B_{i} \backslash I$. Indeed, then we can modify it on a neighborhood of $I$ in $\bigcup_{i} \partial B_{i}$, defining a global smooth vector field, without affecting the requirements (2.2) and (2.3). See Figure 1.
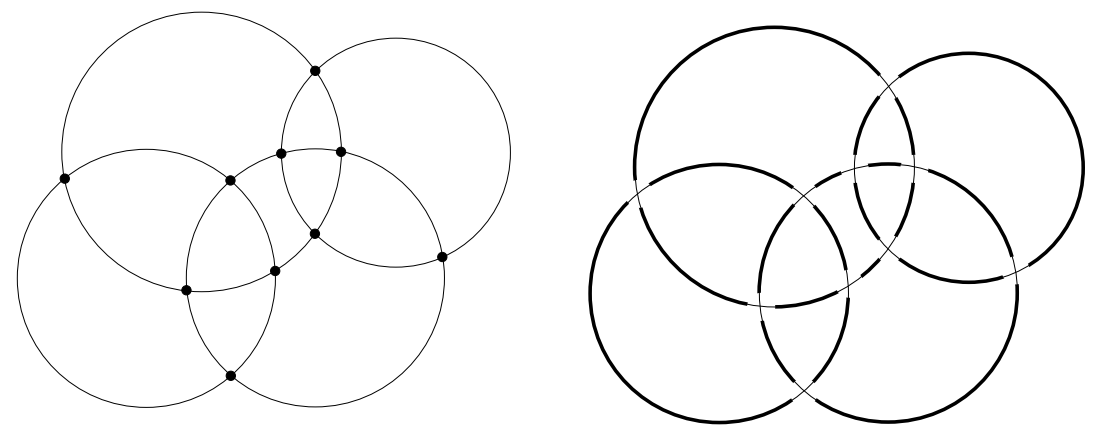

Figure 1. We represent schematically the procedure used to construct the vector field of Lemma 2.5. $V_{m}$ is initially defined outside the finite set of points $I$ which is marked thicker in the drawing on the left. Then we keep $V_{m}$ fixed on the set which is thick on the right, and modify it near the crossings to obtain the final vector field.

We now find $V_{m}$ as above. From Lemma 2.4 it follows that

$$
\sum_{i} \chi_{\partial B_{i}} V \cdot n_{B_{i}} \in L^{p}\left(\bigcup_{i} \partial B_{i}\right)
$$

and has integral in $2 \pi \mathbb{Z}$. Therefore we can take its mollification as a definition of the normal component of $V_{m}$, automatically satisfying (2.2) by the properties of the mollification. Then we can mollify the component of $V$ parallel to $\bigcup \partial B_{i}$, and take the resulting function as the parallel component of $V_{m}$, thereby verifying (2.3) too.

Lemma 2.6. Suppose $\mathscr{B}_{n}$ are families of finitely many balls which cover $B^{2}$ such that each point is not covered more than $C$ times and

$$
\max _{B \in \mathcal{B}_{n}}(\operatorname{diam} B) \rightarrow 0 \quad(n \rightarrow \infty)
$$

Then there holds

$$
\sum_{B \in \mathscr{B}_{n}}\|V-\bar{V}\|_{L^{p}(B)} \rightarrow 0 \quad(n \rightarrow \infty) .
$$

Proof. We take a smooth approximant $W=W_{\varepsilon}$ such that

$$
\|V-W\|_{L^{p}\left(B^{2}\right)} \leq \varepsilon / 4 C .
$$


Then, we can use Poincaré's inequality

$$
\|W-\bar{W}\|_{L^{p}(B)} \leq C r_{B}^{1 / p}\|\nabla W\|_{L^{p}(B)},
$$

and for $n$ big enough there will hold

$$
\sum_{B \in B_{n}}\|W-\bar{W}\|_{L^{p}(B)} \leq \varepsilon / 2 .
$$

Putting together the above two estimates, we obtain

$$
\begin{aligned}
& \sum_{B \in B_{n}}\|V-\bar{V}\|_{L^{p}(B)} \leq \sum_{B \in B_{n}}\|V-W\|_{L^{p}(B)}+\sum_{B \in B_{n}}\|W-\bar{W}\|_{L^{p}(B)} \\
& +\sum_{B \in B_{n}}\|\bar{V}-\bar{W}\|_{L^{p}(B)} \\
& \leq 2 \sum_{B \in B_{n}}\|V-W\|_{L^{p}(B)}+\varepsilon / 2 \\
& \leq 2 C\|V-W\|_{L^{p}\left(B^{2}\right)}+\varepsilon / 2 \\
& \leq \varepsilon,
\end{aligned}
$$

as wanted.

We now distinguish the balls $B_{i}$ based on the value of $\int_{\partial B_{i}} V \cdot n_{B_{i}} d \mathscr{H}^{1}$ : we call $B_{i}$ a good ball in case the integral is zero, and a bad ball in case it is in $2 \pi \mathbb{Z} \backslash\{0\}$.

Lemma 2.7. There exists a constant $C>0$ such that if we have a cover as in Lemma 2.2 with radii not greater than $r:=\varepsilon$, then the number of bad balls satisfies the following estimate:

$$
\#(\text { bad balls }) \leq C \varepsilon^{p-2}\|V\|_{L^{p}}^{p} .
$$

Proof. For a bad ball $B$ we have

$$
1 \leq\left|\int_{\partial B} V \cdot n_{B} d \mathscr{H}^{1}\right|
$$

whence we deduce successively

$$
1 \leq C \varepsilon^{p-1} \int_{\partial B}\left|V \cdot n_{B}\right|^{p} d \mathscr{H}^{1}
$$

and (by summing and using Lemma 2.2)

$$
\#(\text { bad balls }) \leq C \varepsilon^{p-1} \sum_{\text {bad } B} \int_{\partial B}\left|V \cdot n_{B}\right|^{p} d \mathscr{H}^{1} \leq C \varepsilon^{p-2}\|V\|_{L^{p}}^{p},
$$

as wanted. 
Remark 2.8. We observe that by Theorem 1.4, on a good ball the normal component

$$
v_{m}-v: \partial B_{i} \rightarrow \mathbb{R}^{2}, \quad v_{m}-v=n_{B_{i}}\left[\left(V_{m}-V\right) \cdot n_{B_{i}}\right]
$$

satisfies $v_{m}-v=\nabla^{\perp} a_{m}$ for some $W^{1, p}$-function $a_{m}: \partial B_{i} \rightarrow \mathbb{R}$.

Remark 2.9 (explanation of the notation). If we associate to the form

$$
\alpha=\alpha_{1} d x+\alpha_{2} d y
$$

the vector field $V_{\alpha}=\left(\alpha_{2},-\alpha_{1}\right)$, then in an orthonormal frame $n_{B_{i}}, t_{B_{i}}$ given by the normal and tangential unit vectors on $\partial B_{i}$ we see that taking the normal projection done on vector fields, corresponds to restricting the associated form $\alpha$, obtaining $i_{\partial B_{i}}^{*} \alpha$ where $i_{\partial B_{i}}: \partial B_{i} \rightarrow \Omega$ is the inclusion. We can explain our notations above by saying that objects arising from restrictions of forms will be denoted by lower case letters.

The following is a well-known result from the theory of elliptic PDEs.

Lemma 2.10. Let $\tilde{a}$ be a function on the boundary of the unit 2-ball $S^{1}$ having zero mean. Consider the harmonic extension $\tilde{A}$ of ã over $B_{1}$ satisfying

$$
\left\{\begin{aligned}
\Delta \tilde{A} & =0, \\
\tilde{A} & =\tilde{a} \text { on } S^{1} .
\end{aligned}\right.
$$

Then the following estimate holds:

$$
\|\nabla \tilde{A}\|_{L^{p}\left(B_{1}\right)} \leq C\|\nabla \tilde{a}\|_{L^{p}\left(S^{1}\right)} .
$$

We will consider $a_{m}^{\prime}$ on the boundary $\partial B$ of a small ball instead of $\tilde{a}$ on $\partial B_{1}$, and obtain a harmonic extended function, denoted by $A_{m}^{\prime}$, satisfying the analogous of (2.10). Taking into account the scaling factors, we then obtain the following estimate analogous to (2.6) on a ball $B_{r}$ of radius $r$ :

$$
\left\|\nabla A_{m}^{\prime}\right\|_{L^{p}\left(B_{r}\right)} \leq C r^{1 / p}\left\|v_{m}-v\right\|_{L^{p}\left(\partial B_{r}\right)} .
$$

We claim that extending $V_{m}:=\nabla^{\perp} A_{m}^{\prime}+\bar{V}$ inside $B_{r}$, we obtain the wanted approximation:

Lemma 2.11. If $B$ is a good ball of radius $\varepsilon$ on whose boundary we have

$$
\left\|V-V_{m}\right\|_{L^{p}(\partial B)}<\varepsilon,
$$

then the extended smooth vector field $V_{m}$ defined as above satisfies on $B$

$$
\left\|V-V_{m}\right\|_{L^{p}(B)} \leq C \varepsilon^{\frac{p-1}{p}}\left\|v_{m}-v\right\|_{L^{p}(\partial B)}+\|V-\bar{V}\|_{L^{p}(B)} .
$$


Proof. We can then write

$$
\left\|V-V_{m}\right\|_{L^{p}(B)} \leq\|V-\bar{V}\|_{L^{p}(B)}+\left\|\nabla^{\perp} A_{m}^{\prime}\right\|_{L^{p}(B)} .
$$

The second term above is estimated as in (2.7), by $C \varepsilon^{1 / p}\left\|v_{m}-v\right\|_{L^{p}(\partial B)}$, and the estimate (2.3) gives then $\varepsilon\left\|v_{m}-v\right\|_{L^{p}(\partial B)}^{p} \leq C \varepsilon^{p-1}$, finishing the proof.

Lemma 2.12. If $B \subset B_{1}^{2}$ is a bad ball of radius $\varepsilon$ and $v_{m}$ is the smooth orthogonal vector field on $\partial B$ related to $V_{m}$ as in Lemma 2.5 and $V_{r}^{\prime}$ is the radial extension

$$
V_{r}^{\prime}(\theta, \rho):=\frac{\varepsilon}{\rho} v_{m}(\theta)
$$

(in polar coordinates centered in the center of $B$ ), then with the notation

$$
V_{r}:=V_{r}^{\prime}-\bar{V},
$$

we have the estimate

$$
\left\|V-V_{r}\right\|_{L^{p}(B)} \leq\|V-\bar{V}\|_{L^{p}(B)}+C \varepsilon .
$$

Proof. There holds

$$
\begin{aligned}
\left\|V-V_{r}\right\|_{L^{p}(B)} & \leq\|V-\bar{V}\|_{L^{p}(B)}+\left\|V_{r}^{\prime}\right\|_{L^{p}(B)}, \\
\left\|V_{r}^{\prime}\right\|_{L^{p}(B)}^{p} & =\int_{0}^{\varepsilon} \int_{0}^{2 \pi}\left(\frac{\varepsilon}{\rho}\right)^{p}\left|v_{m}(\theta)\right|^{p} d \theta \rho d \rho=C \varepsilon^{2}\left\|v_{m}\right\|_{L^{p}(\partial B)}^{p} .
\end{aligned}
$$

From (2.1), (2.3) and the last equality above we conclude that $\left\|V_{r}^{\prime}\right\|_{L^{p}(B)}^{p} \leq C \varepsilon^{p}$, as wanted.

\subsection{End of proof of Proposition 2.1}

\section{Application of Lemmas 2.11 and 2.12}

We will use the results of Section 2.2 in order to achieve a first global approximation $V_{1}$ of $V$. We again start with the ball $B_{1}$, where we will use Lemma 2.11 or 2.12 , respectively when $B_{1}$ is a good or a bad ball. The new vector field $V_{1}$ obtained by replacing $V$ with the so obtained local approximant on $B_{1}$ satisfies the following properties:

- Good approximation of $\boldsymbol{V}$ on $\boldsymbol{B}_{\mathbf{1}}$ : The approximation error in $L^{p}$-norm on the ball $B_{1}$ is bounded above by $C \varepsilon^{1 / p}+\|V-\bar{V}\|_{L^{p}\left(B_{1}\right)}$.

- Controlled behavior on the boundary: The extension inside $B_{1}$ is equal to $\nabla^{\perp} A_{m}^{\prime}+\bar{V}$ on the boundaries of the balls $B_{i}$, and in particular it has degree equal either to the one of $V_{m}$ or to zero on any of the boundaries of the 
domains $\Omega$ of Lemma 2.5. Indeed, $A_{m}^{\prime}$ is smooth, so $\left.V_{m}\right|_{B_{1}}$ will have divergence either zero (for good balls) or a Dirac mass in the center of $B_{1}$ (for bad balls), while on $B_{1} \backslash \bigcup_{i} \partial B_{i}, V_{m}=V_{1}$. Therefore $V_{1}$ also has the properties stated in Lemma 2.5.

This allows us to apply iteratively the aforementioned construction for the balls $B_{j}, j=2, \ldots, N$, in order to further modify $V_{1}$. We obtain successively approximants $V_{2}, \ldots, V_{N}$ according to Lemmas $2.11,2.12$, and we are able to continue ensuring the smallness condition $\left\|V-V_{m}\right\|_{L^{p}\left(\partial B_{j}\right)}$.

Lemma 2.13. For each $\bar{\varepsilon}>0$ there exist a radius bound $\varepsilon$ and an approximation error bound $\varepsilon_{m}$ (in Lemma 2.5) such that the approximant $V_{N}$ constructed above satisfies

$$
\left\|V-V_{N}\right\|_{L^{p}\left(B^{2}\right)} \leq \bar{\varepsilon} .
$$

Proof. Since in Lemma 2.2 the balls $B_{\frac{3}{8}} r_{i}\left(x_{i}\right)$ are disjoint, we see that no point is covered by more than $\bar{C}$ balls $B_{i}$, where $\bar{C}$ is a geometric packing constant depending on our domain $\Omega$. Therefore in our construction we modify our initial $V$ at most $\bar{C}$ times at each point. This induces a factor $\bar{C}$ in our estimates.

By Lemmas 2.11 and 2.12 we can estimate

$$
\begin{gathered}
\left\|V-V_{N}\right\|_{L^{p}\left(B^{2}\right) \leq} \leq \bar{C} \sum_{\operatorname{good} B}\left[C \varepsilon^{\frac{p-1}{p}}\left\|v_{m}-v\right\|_{L^{p}(\partial B)}+\|V-\bar{V}\|_{L^{p}(B)}\right] \\
+\bar{C} \sum_{\operatorname{bad} B}\left[\|V-\bar{V}\|_{L^{p}(B)}+C \varepsilon\right] \\
=\bar{C} \sum_{\text {all } B}\|V-\bar{V}\|_{L^{p}(B)}+C \varepsilon \#(\text { bad balls })+C \varepsilon^{\frac{p-1}{p}} \varepsilon_{m} .
\end{gathered}
$$

Consider now the expression in the last row above: the first term converges to zero by Lemma 2.6, and the last one is small for $\varepsilon_{m}$ small. The middle term can be estimated using Lemma 2.7 and has thus a bound of the form $C \varepsilon^{p-1}\|V\|_{L^{p}}^{p}$. Since $p>1$ and $V \in L^{p}$, also this term is small for $\varepsilon$ small.

\section{Smoothing on the boundary}

The preceding iteration procedure gives us an $L^{p}$-approximant with error $C \varepsilon$ if the radius $r$ of the balls was chosen to be equal to $\varepsilon$. Moreover it is easy to verify that

$$
\operatorname{div} V_{N}=\sum_{i=1}^{N} \delta_{x_{i}} \int_{\partial B_{i}} V_{m}, \quad \text { locally outside } \bigcup_{i} \partial B_{i},
$$


where $x_{i}$ is the center of $B_{i}$. The resulting vector field $V_{N}$ is however not in $\mathcal{V}_{R}$ : for instance, it is not smooth on all of $\bigcup_{i} \partial B_{i}$. We will thus smoothen $V_{N}$ as follows. We observe that locally near $\bigcup_{i} \partial B_{i}$ on $B^{2} \backslash \bigcup_{i} \partial B_{i}, V_{N}$ is represented as $\nabla^{\perp} A_{i}:=\nabla^{\perp} A_{i}^{\prime}+\bar{V}_{i}$, where $A_{i}^{\prime}$ is smooth and $\bar{V}_{i}$ is a constant equal to the average of $V$ on a particular $B_{i}$. We can take an open cover by small balls of a neighborhood of $\bigcup_{i} \partial B_{i}$, then mollify the functions $A_{i}$ inside each of these small balls, then use a partition of unity to patch the mollifications into a single smooth function $A_{\varepsilon}$, introducing an error of less than $\varepsilon$ in $L^{p}$-norm. Then we can safely define $V_{\varepsilon}:=\nabla^{\perp} A_{\varepsilon}$.

\section{Proof of Main Theorem 1}

Proof. We first show how to deduce the second part of Main Theorem 1 from Proposition 2.1.

The main idea is that, by Proposition 2.1, we can take a sequence $V_{n} \stackrel{L^{p}}{\longrightarrow} V$ which belongs to $\mathcal{V}_{R}$ and construct maps $u_{n}$ such that $V_{n}=\nabla^{\perp} u_{n}$, and they will be constrained to converge to a $u$ with the wanted property $\nabla^{\perp} u=V$. We remark that if $V_{n}$ is smooth and divergence-free outside a discrete set $\Sigma$, then $V_{n}^{\perp}$ is locally holomorphic, and the fact that the divergence around any point of $\Sigma$ is a Dirac mass with coefficient in $2 \pi \mathbb{Z}$ translates into saying that $V_{n}^{\perp}$ has degree equal to that coefficient around that point. Consider the divisor $D$ supported on $\Sigma$ with residue corresponding to the divergence of $V_{n}$. In complex notation $V_{n}^{\perp}$ becomes a meromorphic function with divisor $D$, so we can take $u_{n}:=\arg V_{n}^{\perp}$, which is well-defined with values in $\mathbb{R} / 2 \pi \mathbb{Z}$ and satisfies $\nabla u_{n}=V_{n}^{\perp}$.

We have thus functions $u_{n} \in W^{1, p}\left(\Omega, S^{1}\right)$ satisfying $V_{n}=\nabla^{\perp} u_{n}$ and therefore

$$
\nabla u_{n} \stackrel{L^{p}}{\longrightarrow} V .
$$

We can change the $u_{n}$ by a constant so that $\frac{1}{|\Omega|} \int_{\Omega} u_{n}=0 \in \mathbb{R} / 2 \pi \mathbb{Z}$. Then by Poincaré's inequality we have that the $u_{n}$ form a $L^{p}$-Cauchy sequence, converging therefore to $\bar{u} \in L^{p}(\Omega, \mathbb{R} / 2 \pi \mathbb{Z})$. After extracting a subsequence

$$
u_{n} \stackrel{W^{1, p}}{\longrightarrow} u \in W^{1, p} .
$$

Since we have a.e.-convergence too, it must hold $u=\bar{u}$ and $\nabla^{\perp} u=V$, as wanted. As above,

$$
u_{n} \stackrel{W^{1, p}}{\longrightarrow} u
$$

and $d\left(u_{n}^{*} \theta\right)$ are finite sums of Dirac masses with integer coefficients. The fact that for $u \in W^{1, p}(\Omega, \mathbb{R} / 2 \pi \mathbb{Z})$ the vector field $\nabla^{\perp} u$ has the properties required in the 
theorem, follows from Theorem 1.5, by taking

$$
I=I_{z}^{u}=\tau\left(u^{-1}(z), 1, \frac{\nabla^{\perp} u(x)}{\left|\nabla^{\perp} u(x)\right|}\right),
$$

for a common regular value $z \in \mathbb{R} / 2 \pi \mathbb{Z}$ of all the $u_{n}$ and of $u$. With this choice, using the coarea formula (observe that in our case $\left|J_{u}\right|=\left|\nabla^{\perp} u\right|$ ), we obtain, for all $f \in C_{c}^{\infty}(\Omega)$,

$$
\begin{aligned}
\int_{\Omega} u^{*} \theta \wedge d f & =\int_{\Omega} \nabla^{\perp} u \cdot \nabla f d x=\int_{S^{1}} d y \int_{u^{-1}(y)}\left\langle d f, \frac{\nabla^{\perp} u}{\left|\nabla^{\perp} u\right|}\right\rangle d \mathscr{H}^{1} \\
& =\int_{S^{1}} I_{y}^{u}(d f) d y=\int_{S^{1}} \partial I_{y}^{u}(f) d y .
\end{aligned}
$$

Similarly we obtain for all $n$ :

$$
\int_{\Omega} u_{n}^{*} \theta \wedge d f=\int_{S^{1}} \partial I_{y}^{u_{n}}(f) d y=2 \pi \partial I_{z}^{u_{n}}(f),
$$

since for functions $u_{n}$ having finitely many singularities, $\partial I_{y}^{u_{n}}(f)$ does not depend on $y$. We have (since $\left.C_{c}^{\infty} \subset\left(W^{1, p}\right)^{*}\right)$

$$
\int_{S^{1}} \partial I_{y}^{u_{n}}(f) d y \rightarrow \int_{S^{1}} \partial I_{y}^{u}(f) d y .
$$

Without loss of generality, recalling Theorem 1.5 we may assume that the integrands on the left converge pointwise at $z$, and that the mass $\mathbb{M}\left(I_{z}^{u}\right)$ is bounded. This proves the condition (1.4) with $I=I_{z}^{u}$, thus finishing the proof.

\subsection{Proof of the second version of the Main Theorem}

Proof. We consider the diffeomorphism $\varphi: \mathbb{R} / 2 \pi \mathbb{Z} \rightarrow S^{1} \subset \mathbb{R}^{2}$ given by $t \mapsto$ $(\cos t, \sin t)$, and then instead of the map $u: \Omega \rightarrow \mathbb{R} / 2 \pi \mathbb{Z}$ obtained in the Main Theorem 1 we take the map $\bar{u}:=\varphi \circ u: \Omega \rightarrow S^{1} \subset \mathbb{R}^{2}$. We then obtain

$$
\nabla \bar{u}=\nabla u \otimes(\nabla \varphi \circ u)=\left(\begin{array}{ll}
-\partial_{1} u \sin u & \partial_{1} u \cos u \\
-\partial_{2} u \sin u & \partial_{2} u \cos u
\end{array}\right),
$$

therefore

$$
\bar{u}_{1} \nabla^{\perp} \bar{u}_{2}-\bar{u}_{2} \nabla^{\perp} \bar{u}_{1}=\cos ^{2} u\left(\begin{array}{c}
-\partial_{2} u \\
\partial_{1} u
\end{array}\right)+\sin ^{2} u\left(\begin{array}{c}
-\partial_{2} u \\
\partial_{1} u
\end{array}\right)=\nabla^{\perp} u .
$$

This proves the wanted identifications, and we only need to prove that if we have $\bar{u} \in W^{1, p}\left(\Omega, S^{1}\right)$, then $\bar{u}_{1} \nabla^{\perp} \bar{u}_{2}-\bar{u}_{2} \nabla^{\perp} \bar{u}_{1} \in L^{p}\left(\Omega, \mathbb{R}^{2}\right)$. This follows using the relation $\bar{u}_{1}^{2}+\bar{u}_{2}^{2}=1$ and its consequence $\bar{u}_{1} \nabla^{\perp} \bar{u}_{1}=-\bar{u}_{2} \nabla^{\perp} \bar{u}_{2}$. We have 
indeed:

$$
\begin{aligned}
\left|\bar{u}_{1} \nabla^{\perp} \bar{u}_{2}-\bar{u}_{2} \nabla^{\perp} \bar{u}_{1}\right|^{2} & =\bar{u}_{1}^{2}\left|\nabla^{\perp} \bar{u}_{2}\right|^{2}-2 \bar{u}_{1} \bar{u}_{2} \nabla^{\perp} \bar{u}_{2} \nabla^{\perp} \bar{u}_{2}+\bar{u}_{2}^{2}\left|\nabla^{\perp} \bar{u}_{1}\right|^{2} \\
& =\left(\bar{u}_{1}^{2}+\bar{u}_{2}^{2}\right)\left|\nabla^{\perp} \bar{u}_{2}\right|^{2}+\left(\bar{u}_{1}^{2}+\bar{u}_{2}^{2}\right)\left|\nabla^{\perp} \bar{u}_{1}\right|^{2} \\
& =\left(\partial_{2} \bar{u}_{2}\right)^{2}+\left(\partial_{1} \bar{u}_{2}\right)^{2}+\left(\partial_{2} \bar{u}_{1}\right)^{2}+\left(\partial_{1} \bar{u}_{1}\right)^{2} \\
& =|\nabla \bar{u}|^{2},
\end{aligned}
$$

and since $u \in W^{1, p}$, this proves the result.

\section{Proof of Proposition 2.2}

Our aim here is to prove the following

Proposition 4.1. Given $r>0$, there exists a cover of $B_{1}^{2}$ by a finite set of balls $\left\{B_{r}\left(y_{1}\right), \ldots, B_{r}\left(y_{N}\right)\right\}$ such that the balls $B_{r / 2}\left(y_{i}\right)$ are disjoint and such that for some constant depending only on $p$ and on the dimension,

$$
\sum_{i=1}^{N} \int_{\partial B_{r}\left(y_{i}\right)}\left|V \cdot n_{B_{r}\left(y_{i}\right)}\right|^{p} d x \leq C_{2, p} r^{-1}\|V\|_{L^{p}\left(B^{2}\right)}^{p},
$$

where $n_{B_{r}\left(y_{i}\right)}$ is the outer unit normal vector to the circle $\partial B_{r}\left(y_{i}\right)$.

Directly form the proof of Proposition 4.1 we can also obtain the more refined result:

Proposition 4.2. Given a real $r>0$, there exists a natural number $N$, a set of centers $\left\{x_{1}, \ldots, x_{N}\right\}$ and a positive measure subset $E \subset[3 / 4 r, r]^{N}$ such that for all $\left(r_{1}, \ldots, r_{N}\right) \in E$ the following hold:

- The balls $\left\{B_{1}, \ldots, B_{N}\right\}$ with $B_{i}=B_{r_{i}}\left(x_{i}\right)$ cover $B^{2}$.

- The smaller balls $B_{\frac{3}{8}} r_{i}\left(x_{i}\right)$ are disjoint.

- For some constant depending only on $p$ and on the dimension, there holds

$$
\sum_{i=1}^{N} \int_{\partial B_{i}}\left|V \cdot n_{B_{i}}\right|^{p} d x \leq C_{2, p} r^{-1}\|V\|_{L^{p}\left(B^{2}\right)}^{p} .
$$

\subsection{Equivalent definition of the pointwise norm of $V$}

$\langle V, \theta\rangle$ for a vector $\theta \in S^{1} \subset \mathbb{R}^{2}$ can be expressed as $|V||\cos \gamma|$ where $\gamma$ is the angle between $\theta$ and $V$. After noting

$$
\int_{S^{1}}|\cos \gamma|^{p} d \theta=: c_{p}
$$


we can write

$$
|V|^{p}=\frac{1}{c_{p}} \int_{S^{1}}|\langle V, \theta\rangle|^{p} d \theta .
$$

We now pass to consider the circle $S_{r}(x)=\partial B_{r}(x)$. Then we can write

$$
\begin{aligned}
\int_{S_{r}(x)} V(y) \cdot n_{B_{r}}(y) d y & =\int_{S_{r}(x)}\left\langle V(y),\left(\frac{y-x}{|y-x|}\right)\right\rangle d y \\
& =\int_{S^{1}}\langle V(x+r \theta), \theta\rangle r d \theta .
\end{aligned}
$$

Given a positive number $r$, a point $x \in \mathbb{R}^{2}$ then belongs to the circle $S_{r}(y)$ exactly for $y \in S_{r}(x)$, and we have by (4.2) that

$$
\begin{aligned}
\int_{S_{r}(x)}\left|V(x) \cdot n_{B_{r}(y)}(x)\right|^{p} d y & =\int_{S_{r}(x)}\left|\left\langle V(x),\left(\frac{x-y}{|x-y|}\right)\right\rangle\right|^{p} d y \\
& =\int_{S^{1}}|\langle V(x), \theta\rangle|^{p} r d \theta \\
& =c_{p} r|V(x)|^{p} .
\end{aligned}
$$

\subsection{Proposition 4.1 and an extension of it}

Proof of Proposition 4.1. We observe that (4.3) can be integrated on $\mathbb{R}^{2}$ (after having extended $V$ by zero outside $B^{2}$ ), to give

$$
\begin{aligned}
c_{p} r \int_{B^{2}}|V(x)|^{p} d x & =c_{p} r \int_{\mathbb{R}^{2}}|V(x)|^{p} d x \\
& =\int_{\mathbb{R}^{2}} \int_{S_{r}(x)}\left|V(x) \cdot n_{B_{r}(y)}(x)\right|^{p} d y d x \\
& =\int_{\mathbb{R}^{2}} \int_{S_{r}(z)}\left|V(x) \cdot n_{B_{r}(z)}(x)\right|^{p} d x d z \\
& =\int_{B_{1+r}^{2}} \int_{S_{r}(z)}\left|V(x) \cdot n_{B_{r}(z)}(x)\right|^{p} d x d z
\end{aligned}
$$

We now define some systems of disjoint balls. We consider a set

$$
S=\left\{x_{1}, \ldots, x_{N}\right\} \subset B_{1+r}^{2} \quad \text { s.t. } \quad\left\{\begin{array}{l}
\min _{1 \leq i \neq j \leq N} d\left(x_{i}, x_{j}\right) \geq r, \\
S \text { is maximal, }
\end{array}\right.
$$

and the corresponding set of translates of the ball $B_{r}(0)$

$$
\S:=S+B_{r}(0)=\left\{\left\{x_{1}+y, \ldots, x_{N}+y\right\}: y \in B_{r}(0)\right\} .
$$


Then 8 covers $B_{1+r}$ (by maximality in the definition of $S$ ) at most $C$ times, where $C$ is a packing number (by the requirement on the mutual distances of elements of $S$ ). We can then bound the integral (4.4) from below as follows:

$$
\begin{aligned}
c_{p} r \int_{B^{2}}|V(x)|^{p} d x & =\int_{B_{1+r}^{2}} \int_{S_{r}(z)}\left|V(x) \cdot n_{B_{r}(z)}(x)\right|^{p} d x d z \\
& \geq \frac{1}{C} \int_{B_{r}}\left(\sum_{i=1}^{N} \int_{S_{r}\left(x_{i}+z\right)}|V \cdot n|^{p} d y\right) d z
\end{aligned}
$$

and it follows that there exists $z \in B_{r}$ such that

$$
\sum_{i=1}^{N} \int_{S_{r}\left(x_{i}+z\right)}|V \cdot n|^{p} d y \leq \frac{C c_{p} r}{\left|B_{r}\right|} \int_{B^{3}}|V|^{p} d x=C_{2, p} r^{-1}\|V\|_{L^{p}\left(B^{2}\right)}^{p} .
$$

This is enough to prove (4.1). Moreover, again by the maximality of $S_{0}$, the balls $\left\{B_{r}\left(x_{i}+z\right)\right\}_{i=1}^{N}$ cover $B_{1}^{2}$, and by the requirement on the distances of the centers in (4.5), the $B_{r / 2}\left(x_{i}+z\right)$ are disjoint, proving Proposition 4.1.

\section{Proof of Proposition 2.3}

Suppose here that we are given a vector field $V \in L^{p}\left(B^{2}, \mathbb{R}^{2}\right)$, for some $p \neq \infty$, such that for some integer multiplicity rectifiable current $I$ we have $\operatorname{div} V=\partial I$. This means more precisely that

$$
\int V \cdot \nabla \phi=\langle I, d \phi\rangle, \quad \text { for all functions } \phi \in C_{0}^{\infty}\left(B^{2}\right) .
$$

Here $\langle I, d \phi\rangle$ refers to the action of the current $I$ on the 1 -form $d \phi$. If $\Omega$ is a piecewise smooth domain, we will also call $\partial \Omega_{t}$ the set $\left\{x: \operatorname{dist}_{\partial \Omega}(x)=t\right\}$. By dist $\partial \Omega$ we here denote the oriented distance from $\partial \Omega$, i.e. the function defined on a small

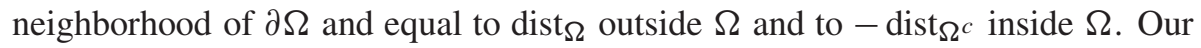
aim in this section is to prove the following

Proposition 5.1. Given a piecewise smooth domain $\Omega \subset B^{2}$, for almost all values $t \in[-\varepsilon, \varepsilon]$ the following properties hold:

- The slice $\left\langle I, \operatorname{dist}_{\partial \Omega}, t\right\rangle$ exists and is a rectifiable 0-current with multiplicity in $2 \pi \mathbb{Z}$.

- The map $\int_{\partial \Omega_{t}} V(y) \cdot n_{t}(y) d \mathscr{H}^{1}(y)$ (where $n_{t}$ is the unit normal to $\left.\partial \Omega_{t}\right)$ is well-defined and coincides with the number $\left\langle I\right.$, $\left.\operatorname{dist}_{\partial \Omega}, t\right\rangle(1) \in 2 \pi \mathbb{Z}$. 
Proof. We consider a family of symmetric mollifiers $\varphi_{\varepsilon}: \mathbb{R} \rightarrow \mathbb{R}^{+}$supported in $[-\varepsilon, \varepsilon]$, and their primitives $\chi_{\varepsilon}(x):=\int_{-\infty}^{x} \varphi_{\varepsilon} d t$. We will consider a non-negative function $g$ which is $C_{c}^{\infty}$-extensions to a neighborhood of $\partial \Omega$ of the constant function equal to 1 on all the sets $\Omega_{t}$ with $t \in[-2 \varepsilon, 2 \varepsilon]$, and we write the current $I$ as $\left(\mathcal{M}_{I}, \theta_{I}, \tau_{I}\right)$, where $\mathcal{M}_{I}$ is a 1-rectifiable set supporting the current $I, \tau_{I}$ is the orienting vector of $I$ and $\theta_{I}$ is the multiplicity of $I$. Then the currents approximating the slice $\langle I, f, t\rangle$ (for some Lipschitz function $f: B^{2} \rightarrow[-2 \varepsilon, 2 \varepsilon]$ ), when it exists, satisfy

$$
\begin{aligned}
I\left\llcorner f^{\#}\left(\varphi_{\varepsilon}(\cdot-t) d \tau\right)(g)\right. & =\int_{\mathcal{M}_{I}}\left\langle\tau_{I}(x), g(x) \varphi_{\varepsilon}(f(x)-t) d f_{x}\right\rangle d \mathscr{H}^{1}(x) \\
& =\int_{\mathcal{M}_{I}}\left\langle\tau_{I}(x), g(x) d\left(\chi_{\varepsilon}(\cdot-t) \circ f\right)_{x}\right\rangle d \mathscr{H}^{1}(x) \\
& =\left\langle I g, d F_{\varepsilon}\right\rangle \quad \text { where } F_{\varepsilon}(x):=\chi_{\varepsilon}\left(f_{\varepsilon}(x)-t\right) \\
& =\left\langle I, d F_{\varepsilon}\right\rangle \quad \text { since } \operatorname{spt} F_{\varepsilon} \subset\{g=1\} \\
& =\int_{\{x:|f(x)-t| \leq \varepsilon\}} V \cdot \nabla F_{\varepsilon} d x^{2} \quad(\text { by }(5.1)) .
\end{aligned}
$$

Now we take $f(x):=\operatorname{dist}_{\partial \Omega}(x)$, obtaining that a.e. on a tubular neighborhood

$$
T(\Omega, 2 \varepsilon):=\bigcup_{-2 \varepsilon \leq t \leq 2 \varepsilon} \partial \Omega_{t},
$$

$\nabla f$ exists, and on each $\partial \Omega_{\tau}=\{f=\tau\}$ it is a.e. equal to the unit normal vector $n_{\tau}$. Therefore we have

$$
\begin{aligned}
\nabla F_{\varepsilon}(x) & =\nabla\left(\chi_{\varepsilon}(\cdot-t) \circ f_{\varepsilon}\right)(x) \\
& =\varphi_{\varepsilon}(\cdot-t) \circ f(x) \nabla f(x)=\left[\varphi_{\varepsilon}(\cdot-t) \circ \operatorname{dist}(x, \partial \Omega)\right] n_{\operatorname{dist}_{\partial \Omega}(x)}
\end{aligned}
$$

and

$$
\begin{aligned}
\int_{\{|f-t| \leq \varepsilon\}} V \cdot \nabla F_{\varepsilon} d x^{2} & =\int_{T(\Omega, 2 \varepsilon)} \varphi_{\varepsilon} \circ \operatorname{dist}_{\partial \Omega_{t}}(x) V(x) \cdot \nabla\left(\operatorname{dist}_{\partial \Omega_{t}}\right)(x) d x^{2} \\
& =\int_{-\varepsilon}^{\varepsilon} \varphi_{\varepsilon}(t)\left(\int_{\partial \Omega_{t}} V \cdot n_{t} d \mathscr{H}^{1}\right) d t .
\end{aligned}
$$

As in the usual theory of slicing, for almost all $t$ the currents $I\left\llcorner f^{\#}\left(\varphi_{\varepsilon}(\cdot-t) d \tau\right)\right.$ converge weakly to the slice $\langle I, f, t\rangle$ as $\varepsilon \rightarrow 0$. Similarly, $V$ being in $L^{p}$, a dominated convergence argument gives also for almost all $\bar{t}$ the convergence

$$
\int_{-\varepsilon}^{\varepsilon} \varphi_{\varepsilon}(\tau-t)\left(\int_{\partial \Omega_{\tau}} V \cdot n_{\partial \Omega_{\tau}} d \mathscr{H}^{1}\right) d t \rightarrow \int_{\partial \Omega_{t}} V \cdot n_{\partial \Omega_{t}} d \mathscr{H}^{1} .
$$


The fact that almost all slices of an integer multiplicity rectifiable current are integer multiplicity rectifiable gives the first point of the proposition, while the second point follows from (5.2) and (5.3).

\section{Further remarks concerning the Main Theorem}

We want first to point out that not all boundaries of rectifiable integral currents $\partial I$ are representable as $u^{*} \theta$ for $u \in W^{1, p}\left(\Omega, S^{1}\right)$, if $p>1$, showing that this case is more subtle than the case $p=1$ treated in Theorem 1.2. To do this, we use the second formulation of the Main Theorem, which says that such $u^{*} \theta$ would then be equal to $V^{\perp}$ for some vector field $V \in L^{p}$ satisfying $\operatorname{div} V=\partial I$. We will show that not all integral currents $I$ have $\partial I$ equal to a divergence of a $L^{p}$-vector field.

Suppose first that we have a vector field $V$ on $B_{\varepsilon}(p)$ satisfying $\operatorname{div} V=\delta_{p}$ (where $\delta_{p}$ is the Dirac mass in $p$ ). Then for almost all $r \in[0, \varepsilon$ [ we have

$$
\int_{\partial B_{r}(p)} V \cdot n_{B_{r}(p)} d \mathscr{H}^{1}=1,
$$

and we see that under the constraint (6.1), the minimal $L^{p}$-mass is achieved by the radial (in polar coordinates around $p$ ) vector field

$$
V_{\min }(\theta, r)=\frac{1}{2 \pi r} \hat{r}
$$

(by a rearrangement argument and by the convexity of the $L^{p}$-norm for $p>1$ ). We therefore obtain (for some geometric constant $C$ )

$$
\|V\|_{L^{p}\left(B_{\varepsilon}(p)\right)}^{p} \geq\left\|V_{\min }\right\|_{L^{p}\left(B_{\varepsilon}(p)\right)}^{p}=C \varepsilon^{2-p} .
$$

We see that such estimate on the norm of $V$ is only dependent on the fact that $(\operatorname{div} V)\left\llcorner B_{\varepsilon}(p)=\delta_{p}\right.$. We can now use a series of inequalities like (6.2) on a series of (disjoint) balls in order to find our counterexample.

Example 6.1. Take a sequence of positive numbers $\left(a_{i}\right)_{i \in \mathbb{N}}$ such that

$$
\begin{gathered}
\sup _{i} a_{i}=\varepsilon, \\
\sum_{i=1}^{\infty} a_{i}=2, \\
\sum_{i=1}^{\infty} a_{i}^{2-p}=+\infty .
\end{gathered}
$$

It is possible to achieve this for any $\varepsilon>0$, since $p>1$. 
Now take a 2-dimensional domain $\Omega$. It is possible to find a series of disjoint balls $B_{i}$ of radii $a_{i}$ for any sequence $a_{i}$ as above, provided that $\varepsilon$ is small enough (because $\mathscr{H}^{1}(\Omega)=\infty$ and for any set $C, \mathscr{H}^{1}(C)>0$ implies $H^{2-p}(C)=\infty$ ). Inside each $B_{i}$ one can insert two disjoint balls $B_{i}^{+}, B_{i}^{-}$of radius $\frac{a_{i}}{2}$. Call $x_{i}^{ \pm}$the center of $B_{i}^{ \pm}$, and consider the current

$$
I=\sum_{i=1}^{\infty}\left[x_{i}^{-}, x_{i}^{+}\right] .
$$

Using the estimate (6.4) and the estimates (6.2) on the disjoint balls $B_{i}^{ \pm}$, we obtain that any vector field satisfying the condition $\operatorname{div} V=\partial I$ must not be in $L^{p}$. By our Main Theorem (second version), we see that none of the currents constructed in this way can possibly have boundary equal to the distributional Jacobian of a map $u \in W^{1, p}\left(\Omega, S^{1}\right)$.

Acknowledgments. I would like to thank Professor Tristan Rivière for introducing me to the topic of this paper and for the many fruitful discussions that we had on the subject.

\section{Bibliography}

[1] G. Alberti, S. Baldo and G. Orlandi, Functions with prescribed singularities, J. Eur. Math. Soc. (JEMS) 5 (2003), 275-311.

[2] F. Bethuel, A characterization of maps in $H^{1}\left(B^{3}, S^{2}\right)$ which can be approximated by smooth maps, Ann. Inst. H. Poincaré Anal. Non Linéaire 7 (1990), 269-286.

[3] F. Bethuel, The approximation problem for Sobolev maps between two manifolds, Acta Math. 167 (1991), 153-206.

[4] F. Bethuel, J.-M. Coron, F. Demengel and F. Hélein, A cohomological criterion for density of smooth maps in Sobolev spaces between two manifolds, in: Nematics (Orsay 1990), NATO Advanced Science Institutes Series C: Mathematical and Physical Sciences 332, Kluwer Academic Publishers, Dordrecht (1991), 15-23.

[5] B. Bojarski, P. Hajłasz and P. Strzelecki, Sard's theorem for mappings in Hölder and Sobolev spaces, Manuscripta Math. 118 (2005), 383-397.

[6] H. Brezis, J.-M. Coron and E. H. Lieb, Harmonic maps with defects, Comm. Math. Phys. 107 (1986), 649-705.

[7] L. de Pascale, The Morse-Sard theorem in Sobolev spaces, Indiana Univ. Math. J. 50 (2001), 1371-1386. 
[8] F. Demengel, Une caractérisation des applications de $W^{1, p}\left(B^{N}, S^{1}\right)$ qui peuvent être approchées par des fonctions régulières, C. R. Math. Acad. Sci. Paris 310 (1990), $553-557$.

[9] A. Figalli, A simple proof of the Morse-Sard theorem in Sobolev spaces, Proc. Amer. Math. Soc. 136 (2008), 3675-3681.

[10] F. Hang and F. Lin, Topology of Sobolev mappings. II, Acta Math. 191 (2003), 55107.

[11] T. Kessel, Singular bundles with $L^{2}$ bounded curvatures, Ph.D. thesis, ETH Zürich, 2008 .

[12] T. Kessel and T. Rivière, Singular bundles with bounded $L^{2}$-curvatures, Boll. Unione Mat. Ital. (9) 1 (2008), 881-901.

[13] J. Malý, D. Swanson and W. P. Ziemer, The co-area formula for Sobolev mappings, Trans. Amer. Math. Soc. 355 (2003), 477-492 (electronic).

Received October 25, 2011; revised March 9, 2012; accepted March 16, 2012.

\section{Author information}

Mircea Petrache, Departement Mathematik, ETH Zürich,

Rämistrasse 101, 8092 Zürich, Switzerland.

E-mail: mircea.petrache@math.ethz.ch 